\title{
Evolution of anticipatory effects mediated by epigenetic changes
}

\author{
Ilkka Kronholm ${ }^{1}$
}

${ }^{1}$ Department of Biological and Environmental Sciences, University of Jyväskylä, FI-40014 Jyväskylä, Finland 
Running Head: Evolution of anticipatory effects

Keywords: between generation plasticity, genetic architecture, phenotypic plasticity, transgenerational plasticity, intergenerational effects

Corresponding author:

Ilkka Kronholm

Department of Biological and Environmental Sciences,

University of Jyväskylä,

P.O. Box 35, FI-40014

Jyväskylä,

Finland

Fax +358 14617239

Email: ilkka.kronholm@jyu.fi 
2

\begin{abstract}
Anticipatory effects mediated by epigenetic changes occur when parents modify the phenotype of their offspring by making epigenetic changes in their gametes guided by information from an environmental cue. To investigate when do anticipatory effects mediated by epigenetic changes evolve in a fluctuating environment, I use an individual based simulation model with explicit genetic architecture. The model allows for the population to respond to environmental changes by evolving within generation plasticity, bet-hedging, or track the environment with genetic adaptation, in addition to the evolution of anticipatory effects. The results show that anticipatory effects evolve when the environmental cue provides reliable information about the environment and the environmental fluctuations span from a few to tens of generations, provided that fitness costs of anticipatory effects are rather low. Moreover, evolution of anticipatory effects is quite robust to different genetic architectures when reliability of the environmental cue is high. Anticipatory effects always give smaller fitness benefits than within generation plasticity, suggesting a possible reason for generally small observed anticipatory effects in empirical studies. The model suggests that empirical studies could focus on taxa that live in environments that have high environmental autocorrelations or environmental cues that reliably predict the environment to test model predictions.
\end{abstract}




\section{${ }_{16}$ Introduction}

Some organisms are able to prime their offspring for future environmental challenges that the offspring are likely

18 to face. These effects have been called anticipatory parental effects, between generation plasticity, induced epigenetic changes, or transgenerational effects in the literature (Jablonka \& Raz, 2009; Marshall \& Uller, 2007; Kronholm, 2017). From now on, I will use the term anticipatory effects following Marshall \& Uller (2007), to focus on the phenotypic consequences and to remain agnostic the about molecular mechanism responsible for the effect. The best examples of anticipatory effects have been observed in plants, and these are often related to defense against pathogens (Rasmann et al., 2012; Slaughter et al., 2012; Luna et al., 2012) or herbivores (Holeski, 2007), but also to abiotic factors such

24 as drought and osmotic stress (Wibowo et al., 2016; Herman et al., 2012) or shading (Galloway \& Etterson, 2007). Anticipatory effects are not restricted to plants, but have been observed in many animals as well. Particularly, there are examples of fish responding to temperature during development in subsequent generations (Salinas \& Munch, 2012; Donelson et al., 2012; Shama \& Wegner, 2014), developmental morphs of water fleas (Agrawal et al., 1999), viral defense, starvation, and life-span in nematodes (Rechavi et al., 2011, 2014; Jobson et al., 2015; Kishimoto et al., 2017; Ivimey-Cook et al., 2020), and diet in fruit flies (Öst et al., 2014).

A general principle in these examples is that the parents experience a particular environment, and as a result they change the phenotype of their offspring in a presumably adaptive manner. Priming the offspring to better cope with this particular environmental challenge, even if the offspring have not yet encountered this environment themselves. For example, offspring may upregulate the expression of possibly costly defence related genes, even if they have not 34 yet encountered a particular pathogen. There are different mechanisms that may accomplish this, such as provisioning of nutrients or hormones to eggs, transfer of antimicrobial peptides or antibodies to the offspring, or epigenetic inheritance. In some cases the mechanistic basis of anticipatory effects based on epigenetic inheritance has been worked out in great detail. The specifics vary by taxon: in the plant Arabidopsis thaliana certain genes are methylated by the RNA-dependent methylation pathway as a response to osmotic stress, and these methylation patterns can be inherited via seed but not by pollen (Wibowo et al., 2016). In the nematode Caenorhabditis elegans environmental stress induces the production of small RNA's that are inherited, and these RNA's subsequently direct chromatin conformation and gene expression changes of their target genes (Rechavi et al., 2014; Rechavi \& Lev, 2017). In fruit flies epigenetic inheritance was dependent on the Polycomb repressive complex silencing machinery (Öst et al., 2014), and small RNA's may be involved in fruit flies as well (Duempelmann et al., 2020).

In ordinary phenotypic plasticity that happens within a single generation, organisms use some sort of environmental cue to inform delevopmental decisions. For instance, changes in day length predict the change of seasons and thus temperature. Conceptually, anticipatory effects closely resemble ordinary phenotypic plasticity that happens within a generation, but the inducing environmental signal has been perceived by the parent rather than the focal organism. In 
48 order for anticipatory effects to occur, there needs to be a genetic pathway already present that can sense, transduce, and respond to an environmental signal, like in normal within generation phenotypic plasticity. Intuitively, the evolution of the capability to use anticipatory effects seems to be contingent on the environmental cue having some predictive power on what the environment will be like in the next generation.

Several previous theoretical studies have investigated the evolution of maternal effects using models based on phenotypic memory, where the phenotype of the parent is partially passed on the to the offspring (Jablonka et al., 1995;

54 Lachmann \& Jablonka, 1996; Hoyle \& Ezard, 2012; Prizak et al., 2014; Kuijper \& Hoyle, 2015; Kuijper \& Johnstone, 2015). Because genes influence the phenotype of the parent and the phenotype is passed partially to offspring these are indirect genetic effects, which can then cascade over multiple generations (Hoyle \& Ezard, 2012; Kuijper \& Hoyle, 2015). While there certainly are some mechanisms where maternal effects can work this way, here I am motivated 58 by the biology of epigenetic changes that can also work in a slightly different manner. In the case of epigenetic inheritance, parents can pass on information about their current environment in the form of chemical modifcations

60 of DNA or associated histone proteins, and this information about the current environment can be different than the current phenotype of the parents. Yet, indirect genetic effects and epigenetic changes can certainly be similar on a conceptual level and lessons learned from indirect genetic effects may apply to anticipatory effects that are mediated by epigenetic changes as well, but this is not completely clear.

Previous studies indicate that the evolution of anticipatory effects is favoured in fluctuating environments (Jablonka et al., 1995; Lachmann \& Jablonka, 1996). However, some models give different results as to what has to be the timescale of environmental fluctuations (Furrow \& Feldman, 2014). Moreover, the evolution of anticipatory effects has been studied in the context of population structure, and moderate levels of gene flow were found to favour the evolution of anticipatory effects (Greenspoon \& Spencer, 2018). It has been suggested that organisms can use different types of strategies to adapt to environmental changes that happen at different time scales (Kristensen et al., 2018). Environmental changes that happen within a generation should favor the evolution of within generation plasticity, slightly longer fluctuations could favour anticipatory effects, and populations should adapt to environmental changes over long periods of time by genetic adaptation. Yet, the exact conditions that the favour the evolution of anticipatory effects in fluctuating environments when alternative strategies are also possible are unclear at the moment. There could

74 be regions of the parameter space where anticipatory effects would evolve if only investigated in isolation but when alternative strategies are also possibly they might have a higher fitness, possibly preventing the evolution of anticipatory effects. Therefore, it is important to investigate the evolution of anticipatory effects in combination with alternative strategies, as done for phenotypic plasticity by Botero et al. (2015). Anticipatory effects may also have fitness costs for the organism, at minimum the products of the genetic pathway responsible anticipatory effects must be synthesized.

Previous models have mainly investigated the evolution of maternal or anticipatory effects using quantitative genetic so framework, where the assumption is that traits are determined by infinitely many small effect loci. While this is likely to 
be a reasonable assumption for many phenotypes, there are certainly examples of large genetic contributions of single loci to the phenotype for a number of different traits, and if genetic architecture is based on a few large effect loci, this can change the observed evolutionary dynamics (Oomen et al., 2020). There could be constrains on the evolution of anticipatory effects if, for example, mutational effects sizes or the number of loci are important factors the evolution of the genetic traits that govern the induction of anticipatory effects.

Furthermore, a recent meta-analysis found that anticipatory effects are mostly weak (Uller et al., 2013). Whether it is the case that conditions for the evolution of anticipatory effects are restrictive such that they are rare in nature, or that those taxa that live in conditions that are favourable for the evolution of anticipatory effects are under represented in studies, or that anticipatory effects are generally to be expected to be low, is not known. By having a clear picture of the conditions where anticipatory effects are expected to evolve, empirical studies can focus on those species that live in such environments.

I ask the question: in what kind of fluctuating environments do anticipatory effects evolve? I mainly focus on invistigating how does the predictability of the environment and the frequency of environmental fluctuations affect the evolution of anticipatory effects. Furthermore, I investigate what effect do costs of phenotypic plasticity and anticipatory effects have on their evolution and ask: does genetic architecture constrain the evolution of anticipatory effects? To answer these questions I use an individual based simulation model adapted and modified from Botero et al. (2015). In the model, each individual has a linear reaction norm that is determined by an explicit genetic architecture. The model allows the population to evolve either reversible phenotypic plasticity where phenotype can be adjusted multiple times per generation, developmental plasticity where phenotype is adjusted only once, increased developmental variation as a type of bet-hedging strategy, or simple genetic adaptation to the environment by tracking the environmental optimum by changing reaction norm intercept. Finally, the population can also evolve epigenetic modification, allowing anticipatory effects, where information from the parents is passed on the the offspring that can adjust their phenotype in early development.

\section{Methods}

\section{Simulation model}

I used an individual based Wright-Fisher model with non-overlapping generations and random mating. The model keeps track of explicit genetics of each individual. Phenotype is determined by multiple loci and environmental effects. Environment changes at different rates and individuals can potentially use an environmental cue to respond by plasticity or by anticipatory parental effects mediated by epigenetic changes. Developmental variation is also partially under genetic control, which makes it possible to evolve increased variation as a bet-hedging type strategy. The model is 
based on a model of phenotypic plasticity used by Botero et al. (2015) with some modifications. The events in the model are shown in Figure 1.

\section{Environmental changes}

114 In the simulation, there are $L$ timesteps in each generation, a total of $t=n_{g} L$ timesteps, where $n_{g}$ is the number of generations. The number of timesteps in each generation was set to 5 . The environment, $E$, changes cyclically according to:

$$
E_{t}=\sin \left(\frac{2 \pi t}{L R}\right)
$$

where $R$ is a parameter that determines how fast the environment changes relative to generation time. Figure $\mathrm{S} 1$ shows how changing $R$ affects the speed of environmental fluctuations. The values of $R$ that were used in the simulations were: $1,3.2,10,31.6,100,316.2,1000,3162.3$, and 10000. In subsequent plots these values are plotted as their base 10 logaritms: $0,0.5,1,1.5,2,2.5,3,3.5$, and 4 .

There is also an environmental cue, $C$, that individuals can potentially use to anticipate changes in the environment. The distribution of the cue is gaussian: $C_{t} \sim \mathrm{N}\left(E_{t} P,(1-P) / 3\right)$, where $P$ is a parameter that determines the predictability of the environment. When $P=1$ the cue predicts the environment perfectly, and when $P=0$, cue is randomly distributed around 0 with standard deviation of $\frac{1}{3}$. Simulations were run for values of $P$ from 0 to 1 at 0.1 intervals.

\section{Genetics, development, and phenotype}

Individuals phenotype, $Z$, is determined by a standard quantitative genetic model with explicit loci. As the model allows for plasticity, the phenotype for individual $i$ is determined by a linear reaction norm

$$
Z_{i}=G_{a, i}+G_{b, i} C_{t}+\epsilon_{i}
$$

where $G_{a}$ is the genotypic value for the intercept, $G_{b}$ is the genotypic value for the slope by which individual changes its phenotype as a reaction to the environmental cue, and $\epsilon$ which is random developmental variation in the phenotype. The random variation in development is distributed as $\epsilon \sim \mathrm{N}\left(0, \sigma_{R}\right)$, where $\sigma_{R}=\sigma_{E}+G_{h, i}$ and $\sigma_{E}$ is the environmental component of random variation and $G_{h}$ the genotypic component of random developmental variation. Thus, the amount of random developmental variation can potentially evolve. There are $l_{a}$ loci that determine the intercept, $l_{b}$ loci that determine the slope, $l_{d}$ loci that determine the propability of plastic adjustment of the phenotype, $l_{h}$ loci that determine the genotypic component of random developmental variation, and $l_{e}$ loci that determine the probability of epigenetic modification of slope loci. Genotypic values are always the sum of individual allelic effects for each 


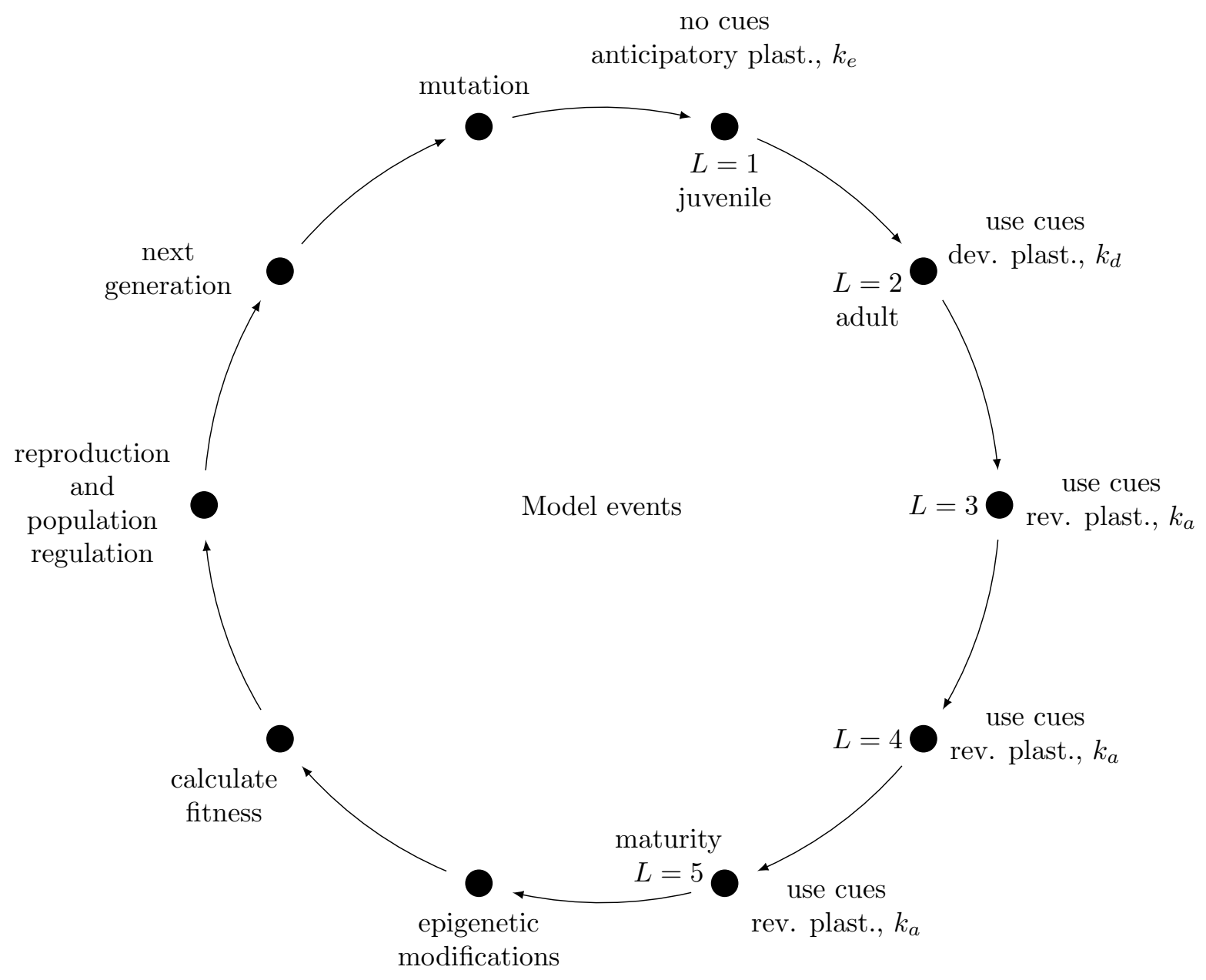

Figure 1: Events in the simulation model. Each individual lives for 5 timesteps. In the first time step individuals can use epigenetic changes inherited from their parents to modify their phenotype and pay a fitness cost of $k_{e}$ for this action. Epigenetic modifications are reset at the end of this step. In the next timestep individuals can use developmental plasticity to adjust their phenotype based on an observed environmental cue, with a fitness cost of $k_{d}$. In timesteps three to five, individuals can use reversible plasticity to further adjust their phenotype based on observed cues, with a fitness of cost of $k_{a}$ for each adjustment. After timestep five, any potential epigenetic modifications are made based on an observed environmental cue from time step five. Then fitness is calculated for each individual, individuals produce offspring weighted by their fitness, population regulation happens, and next generation is formed. Then new mutations are produced in those individuals and the cycle starts again. 
locus. However, genotypic values for adjustment probability, $G_{d}$, and probability of epigenetic modification, $G_{e}$, were

restricted between 0 and 1 . The genotypic values for random developmental variation, $G_{h}$, were restricted to between 0 and 3. The number of loci for each category was set to 10. At the start of the simulation, a genetic map was randomly generated, with all loci distributed randomly and uniformly across $n_{c}$ linkage groups (chromosomes).

Individuals live for five timesteps, during the first stage an individual has a chance to adjust its phenotype based on epigenetic modifications received from parents, but it cannot yet use environmental cues on its own. The anticipatory effect, $G_{b}^{*} C_{t-1}$ is calculated over those slope loci that are epigenetically modified. Modified alleles are encoded as complex numbers with a real part that is the allelic effect and the imaginary part that is the cue experienced by the parents in timestep $t-1$. In the second life stage individuals can use developmental plasticity according to equation 2. In the third, fourth, and fifth life stage individuals can use reversible plasticity to adjust their phenotype based on environmental cues with a probability of $G_{d}$ at each step. In the fifth and final life stage, individuals epigenetically modify all of their slope alleles with a probability of $G_{e}$. If an individuals modifies its alleles it modifies all of them setting the modification to the value of $C_{t}$.

\section{Fitness and selection}

Fitness for each individual over its entire life span is calculated as sum of phenotypic deviations from the environmental 154 optimum at each life stage:

$$
W_{i}=\exp \left(-\tau \sum_{t=1}^{L}\left|E_{t}-Z_{i, t}\right|\right)-n_{e} k_{e}-n_{d} k_{d}-n_{a} k_{a}
$$

where $\tau$ is a parameter for intensity of selection which was set to $0.25, k_{e}$ is the fitness cost of adjusting the phenotype using epigenetic modifications, $n_{e} \in\{0,1\}$ is the number of such adjustments, $k_{d}$ is the fitness cost for adjusting the phenotype by developmental plasticity, $n_{d} \in\{0,1\}$ is the number of such adjustments, $k_{a}$ is the fitness cost for adjusting the phenotype by reversible phenotypic plasticity, and $n_{a} \in\{0,1,2,3\}$ is the number of such adjustments. Any negative values for fitness due to costs of phenotypic plasticity were set to 0 .

\section{Reproduction and population regulation} $N_{R}$ is the number of offspring produced on average by a perfectly adapted individual (that has a relative fitness of 1). the other parent is picked randomly from the population weighted by their fitness. Gametes are produced by meiosis and the number of cross-overs for each pair of homologous chromosomes has a distribution of $n_{x o} \sim$ Pois $(\lambda)+1$, so that the minimum number of cross-overs is always 1 . The $\lambda$ parameter was set to 0.56 , which corresponds to 1.56 
cross-overs for each pair of homologous chromosomes in meiosis. This is the number of chiasmata per chromosome pair that on average is observed across eukaryotes (Otto \& Lenormand, 2002). Cross-over positions are drawn from a uniform distribution along the chromosome, with no interference. If the total number of offspring produced by all individuals was larger the population carrying capacity, $K$, then offspring were removed randomly until $K$ offspring remained.

\section{Mutation}

After the next generation of individuals has been produced, mutations are generated. Each locus has a per locus mutation rate, $\mu$. Since there are $2 N l$ alleles in a population, the mean number of mutations per generation is $2 N l \mu$. Each mutation generates a new allele and the allelic effect is drawn from a gaussian distribution, $\alpha \sim \mathrm{N}\left(0, \sigma_{\alpha}\right)$. Standard deviation of mutational effects was set to 1 . Mutational variance can be calculated as $\sigma_{m}^{2}=2 \mu l \sigma_{\alpha}^{2}$, and mutational heritability by standardizing mutational variance with environmental variance, $h_{m}^{2}=\sigma_{m}^{2} / \sigma_{E}^{2}$. Substituting values used in the simulation to these equations leads to a mutational heritability of 0.2 for the reaction norm intercept or slope. While this value is one to two orders of magnitude higher than typical mutational heritabilities that have been measured experimentally (Halligan \& Keightley, 2009; Durand et al., 2010; Lynch \& Walsh, 1998). The per locus mutation rate used in the simulation, $\mu=10^{-4}$, is much higher than realistic mutation rates are. However, these values of mutational parameters were used because of the relatively small population sizes that could used in the simulation without extending the computational time beyond feasibility.

Table 1: Parameters used in the simulation model.

\begin{tabular}{lll}
\hline Parameter & Explanation & Value \\
\hline$L$ & Number of timesteps in each generation & 5 \\
$n_{g}$ & Number of generations & 3000 or 1500 \\
$R$ & Rate of environmental change relative to generation time & varied \\
$P$ & Prepictability of the environment & varied \\
$\sigma_{E}$ & Standard deviation of random environmental variation & 0.1 \\
$l_{a}$ & Number of loci for intercept effect & 10 \\
$l_{b}$ & Number of loci for slope effect & 10 \\
$l_{d}$ & Number of loci for probability of plastic adjustment & 10 \\
$l_{e}$ & Number of loci for probability of epigenetic modification & 10 \\
$l_{h}$ & Number of loci for genetic component of developmental variation & 10 \\
$n_{c}$ & Number of linkage groups & 4 \\
$\tau$ & Intensity of selection & 0.25 \\
$k_{e}$ & Fitness cost of phenotypic adjustment by epigenetics & varied \\
$k_{d}$ & Fitness cost of developmental plasticity & varied \\
$k_{a}$ & Fitness cost of reversible plasticity & varied \\
$N_{R}$ & Mean number of offspring produced by an individual & 5 \\
$K$ & Population carrying capacity & 5000 \\
$n_{x o}$ & Mean number of cross-overs between homologous chromosomes & 1.56 \\
$\mu$ & Mutation rate per locus per generation & $10^{-4}$ \\
$\sigma_{\alpha}$ & Standard deviation of mutational effects & 1 \\
\hline
\end{tabular}




\section{Simulations and data analysis}

Simulations were written in R (R Core Team, 2019). Replicate simulations were ran in parallel on a computer supercluster utilizing the 'snow' package. To examine in which conditions anticipatory effects evolve, I ran 100 replicate simulations for each combination of $R$ and $P$. Costs of plasticity were set to $k_{d}=k_{e}=0.02$ and $k_{a}=0.01$, which correspond to values used by Botero et al. (2015) for the costs of developmental and reversible plasticity. Each replicate simulation lasted for 3000 generations. After these simulations it was clear that 3000 generations was more than enough for the populations to adapt, so in subsequent simulations 1500 generations were used to reduce computational time. All simulation parameters are shown in table 1. Then I tested how do costs of plasticity affect the strategy populations use to adapt. I ran simulations where there were no costs of phenotypic plasticity or anticipatory effects, $k_{d}=k_{e}=k_{a}=0$ and simulations where the costs were twice as high as in the the original simulations $k_{d}=k_{e}=0.04$ and $k_{a}=0.02$.

\section{Sensitivity analysis}

To examine if the results were robust to different genetic architectures, I ran a sensitivity analysis of the simulation. 100 replicate simulations for each parameter combination of $P$ and $R$ were run. For each replicate, random values were drawn for parameters $\sigma_{E}$, number of different loci, linkage groups, mutation rate, and $\sigma_{\alpha}$. Costs of plasticity were $k_{d}=k_{e}=0.02$ and $k_{a}=0.01$. Other parameters were the same as in previous simulations (Table 1). Values were drawn from following uniform distributions: $\sigma_{E} \sim \mathrm{U}(0.01,0.5), \mu \sim \mathrm{U}\left(10^{-6}, 10^{-3}\right), \sigma_{\alpha} \sim \mathrm{U}(0.01,2)$, number of each loci of each type was randomly selected from $[4,25]$ and number of linkage groups from $[1,10]$. Loci were distributed evenly across the linkage groups, and the remainder was always assigned to linkage group 1.

\section{Data availability}

An R package implementing the simulations is available on github: https://github.com/ikron/indsim.

\section{Results}

\section{Phenotypic plasticity}

When costs of plasticity were $k_{e}=k_{d}=0.02$ and $k_{a}=0.01$ some sort of plasticity consistently evolved when rate of environmental change, $R$, was less than 100 and environmental predictability, $P$, was 0.3 or greater (Figure 2). Reversible plasticity (Figure S2) evolves when rate of environmental change is fast, that is when R is 10 or smaller. Higher values of $R$ favoured the evolution of developmental plasticity (Figure S3), thus when environmental change is slow enough the costs of reversible plasticity outweigh its fitness benefits. Developmental plasticity evolved in some 


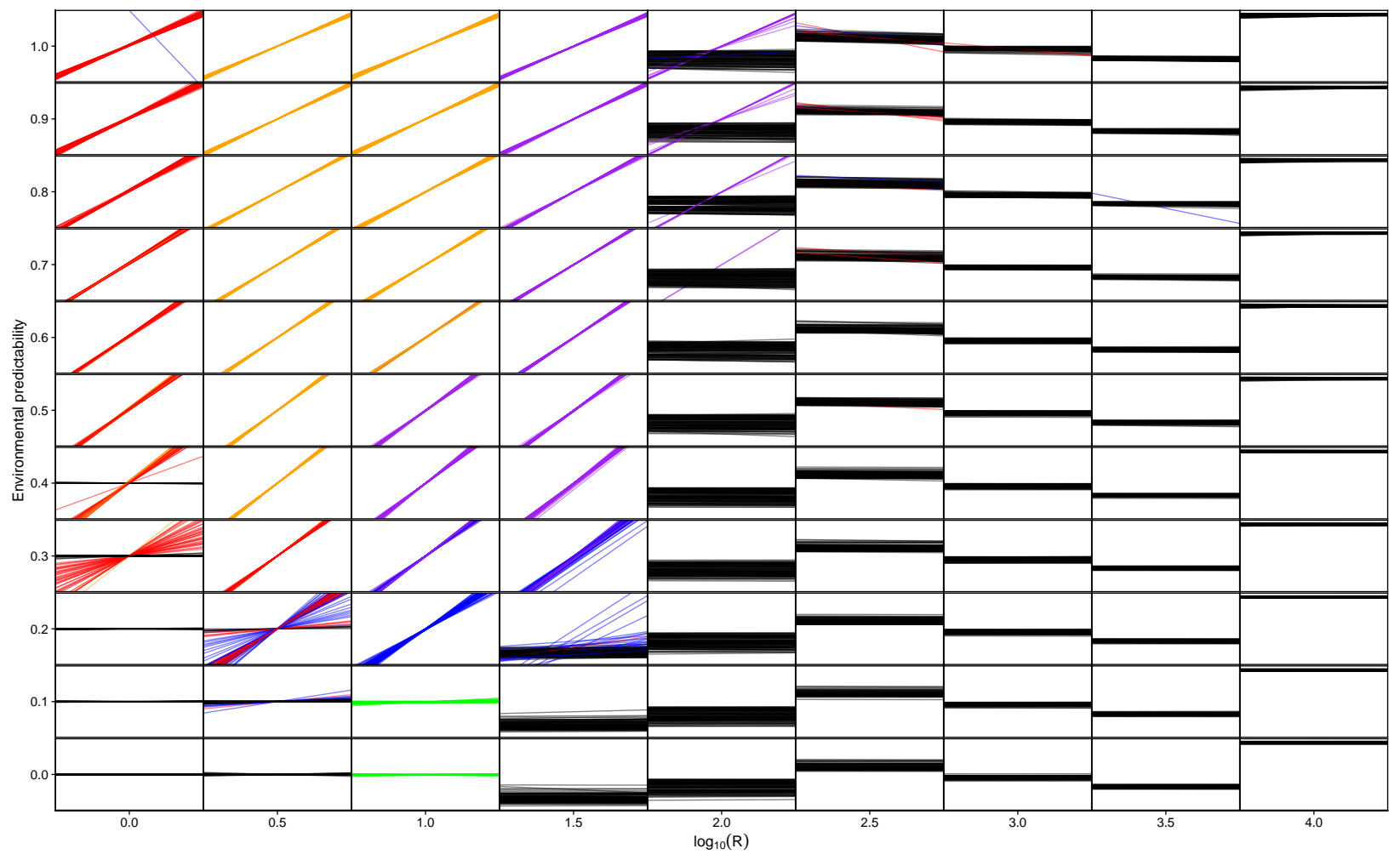

Figure 2: Simulation results for $k_{e}=k_{d}=0.02$ and $k_{a}=0.01$. Each reaction norm is the population mean for one replicate simulation and 100 replicates were performed for each combination of $P$ and $R$. Red colour shows reaction norms, where the population evolved reversible plasticity $\left(G_{b}>0.1\right.$ or $G_{b}<-0.1$, and $\left.G_{d}>0.1\right)$. Orange colour shows reaction norms where reversible and anticipatory effects evolved (in addition $G_{e}>0.1$ ), blue colour indicates that only developmental plasticity evolved $\left(G_{b}>0.1\right.$ or $G_{b}<-0.1$, and $\left.G_{d} \leq 0.1\right)$, purple colour indicates that both developmental plasticity and anticipatory effects evolved (in addition $G_{e}>0.1$ ), green colour indicates that increased developmental variation evolved $\left(G_{h}>0.1\right)$, and black colour that there was no major change in reaction norm slope $\left(-0.1 \leq G_{b} \leq 0.1\right)$. Note the logarithmic scale for $R$.

replicates when $R$ was 100 and predictability high, but slower environmental change did no longer favour the evolution plasticity. Instead the population tracked the moving environmental optimum by changing the reaction norm intercept (Figure S4). When environmental change was unpredictable $(P<0.3)$ and fast $(R=1)$, the reaction norm of the population remained flat with an intercept of 0 (Figure 2). This represents a conservative strategy where individuals are just tolerating environmental changes. However, when environmental changes were slower $(R=10)$ but still unpredictable, the population evolved increased developmental variation (Figure S5). This represents a bet-hedging strategy where individuals are increasing their geometric mean fitness by producing offspring with different phenotypes in order for some of them to be adaptated to the unpredictable environment. Overall the results regarding plasticity are very similar as those obtained by Botero et al. (2015), despite some differences in the genetic architecture of the traits. Here the conditions favouring the evolution of a diversifying bet-hedging strategy are more restrictive than in Botero et al. (2015), but in their model it was possible to evolve a phenotype that produced two distinct morphs, rather than only increased developmental variation as here. 


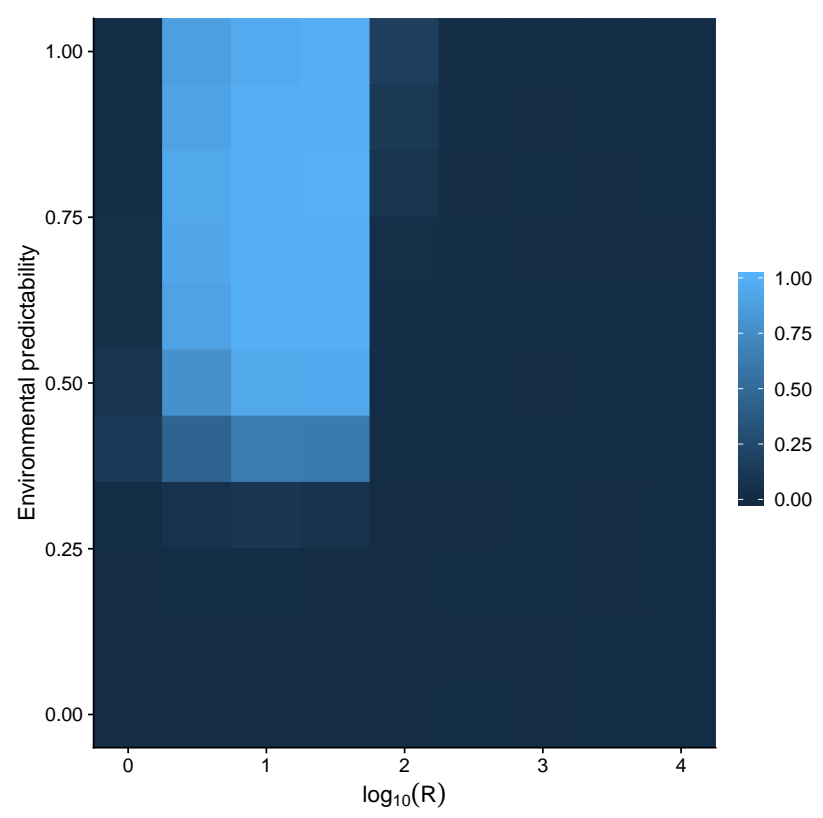

Figure 3: Heat map for mean probability of epigenetic modification from 100 replicate simulations with each combination of $P$ and $R$. Costs of plasticity were $k_{e}=k_{d}=0.02$ and $k_{a}=0.01$.

\section{Anticipatory effects}

Anticipatory effects mediated by epigenetic changes evolved consistently when the environment did not change too fast or too slow, $1<R<100$, and environmental predictability was 0.4 or larger (Figure 2). Very fast environmental changes do not favour the evolution of anticipatory effects, because the environment can change between when the effects are induced in the parents and by the the time offspring develop. Very slow environmental changes also do not favour anticipatory effects as the fitness costs of anticipatory effects do not outweigh their benefits. Finally, the environment has to be rather predictable, which is understandable, since not being able to predict what the offspring environment will be can result in maladaptive anticipatory effects. Anticipatory effects evolved together with either reversible plasticity (Figure S6) when $3.2 \leq R \leq 10$, and together with developmental plasticity (Figure S7) when $10 \leq R \leq 32$

The probability of individuals using epigenetic modifications to mediate anticipatory effects was highest when environmental predictability was more than 0.5 and when $10 \leq R<100$ (Figure 3). Lower values of of $P$ and $R$ gave slightly lower probabilities of epigenetic modification, until they epigenetic modification no longer happened.

Anticipatory effects increased the final fitness the population could achieve. For example, when $R=31.6$ and $P=0.8$ the $95 \%$ quantiles for geometric mean fitness for the last 500 generations in the simulation was $0.72[0.6,0.74]$ for populations where evolution of anticipatory effects was impossible and $0.76[0.6,0.77]$ for a population where anticipatory effects could freely evolve. While this difference is small in absolute terms, a relative fitness of 1.05 in favour of anticipatory effects is more than enough to drive their evolution. 


\section{Costs of plasticity and anticipatory effects}

To test whether the results were robust to genetic architecture of the phenotype, I ran a sensitivity analysis where fitness costs were assumed to be zero, reversible phenotypic plasticity with anticipatory effects was very common throuhout the parameter space (Figure 4A). Clearly if there is no cost anticipatory effects or plasticity, they should be very common, even if environmental cues predict the environment poorly. In figure 4A there is a strip at $R=316.2$ where no anticipatory effects evolve but they seem to evolve at higher values of $R$. This can actually be explained by dynamic alternation of plasticity and genetic assimilation. When $R>100$ and $P>0.3$ environment changes slowly, so that the population tracks the environment mainly by genetic adaptation. However, when there are no costs of plasticity, the population evolves transient plasticity and anticipatory effects during those periods that the environment zero, only to depart from zero again when the environment start moving again (Figure S8). These type of dynamics have been observed before in models of plasticity and maternal effects, where there is an extraordinary environmental change that the population has to adapt to (Lande, 2009; Hoyle \& Ezard, 2012). Plastic and anticipatory effects help speed up adaptation. So this region of parameter space is characterized by transient anticipatory effects and genetic assimilation. Evolutionary changes tend to happen in reaction norm slope, the probability of epigenetic modification changes occasionally, but evolves to moderate or high value. The fitness benefits the population gains from these transient changes in plasticity are small, as any costs of plasticity prevented this type dynamic from occurring (Figure 4). The strip at $R=316.2$ is due to environmental fluctuation being in a phase where genetic assimilation has happened and slope is zero in these populations (Figure S8A).

However, there was a region of parameter space where anticipatory effects did not evolve even no costs. If $P=0$ anticipatory effects did not evolve, or if $R \geq 100$ and $P \leq 0.3$ no plasticity or anticipatory effects evolved (Figure 4A). In this region of parameter space anticipatory effects are likely to be either neutral or possibly deleterious as preparing offspring to a wrong environment can be maladaptive. Increasing fitness costs of epigenetic modification reduces the range where anticipatory effects evolve (Figure 4B) and when both plasticity and epigenetic modification have high enough costs, only reversible or developmental plasticity evolves (Figure 4C).

\section{Influence of genetic architecture}

parameters describing the genetic architecture of the population were randomly selected. The results of sensitivity analysis show that genetic architecture does influence to outcome of the simulations, but similar broad patterns are still recovered as with the initial simulations (Figure 5). Anticipatory effects do evolve, but now higher environmental predictability is generally required, $P \geq 0.6$. Moreover, anticipatory effects now don't evolve as certainly as in the 

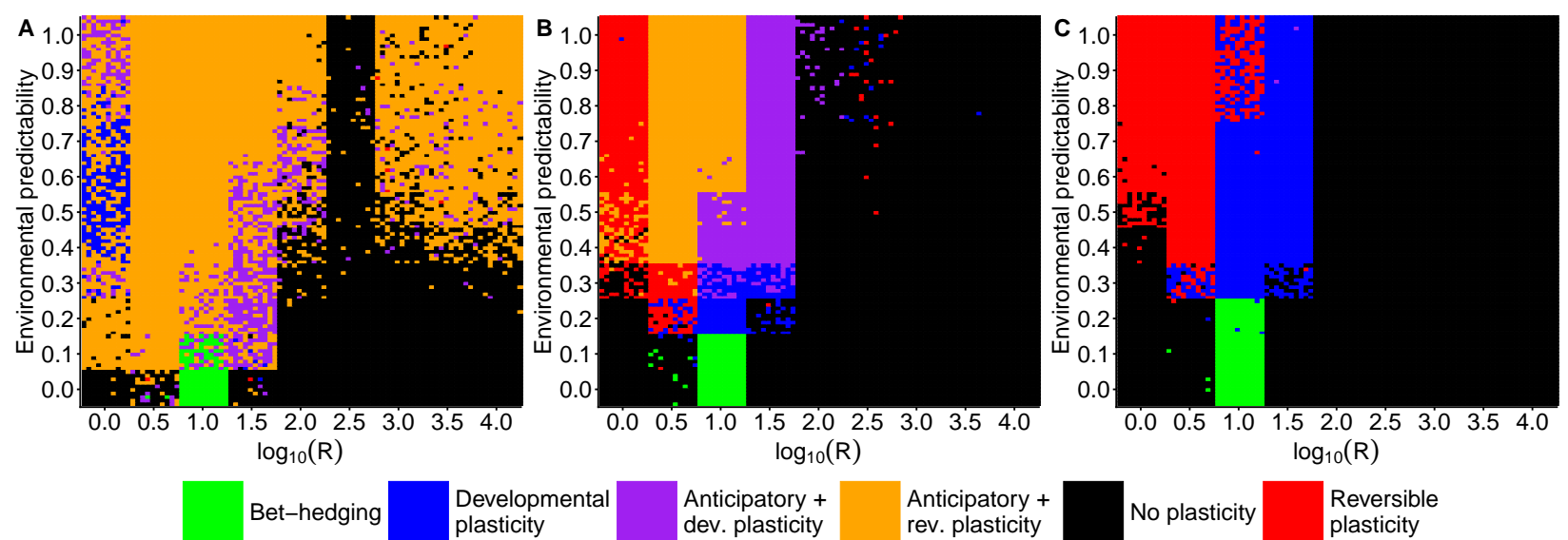

Figure 4: Simulation results with different costs for phenotypic plasticity and anticipatory effects. For each parameter combination of $P$ and $R, 100$ replicate simulations are represented as coloured tiles, with colors corresponding to different strategies evolved in that replicate. Costs are: A) $\left.k_{e}=k_{d}=k_{a}=0 \mathrm{~B}\right) k_{e}=k_{d}=0.02$ and $k_{a}=0.01 \mathrm{C}$ ) $k_{e}=k_{d}=0.04$ and $k_{a}=0.02$.

original simulation even if plasticity evolves when $3.2 \leq R \leq 31.6$ (Figure 5). Since the fitness benefits of anticipatory effects are smaller then within generation plasticity, sometimes genetic architecture does not allow fine tuning of the anticipatory effects, which is seen especially in lower values of environmental predictability where lower probability of epigenetic modification evolves (Figure 3). In some cases the population goes extinct because there is not enough genetic variation for adaptation, but this was not common. In the sensitivity analyses the parameter space where increased developmental variation could evolve was actually larger, reflecting that if the environmental component of random variation was small there was more opportunity to sometimes evolve increased developmental variation (Figure $5)$.

\section{Discussion}

The results reported here largely agree with previous modeling results concerning maternal effects in phenotypic memory type of models, in that anticipatory effects can evolve as long as environmental cues can predict the environment of the offspring to some degree (Hoyle \& Ezard, 2012; Ezard et al., 2014; Kuijper \& Hoyle, 2015; Kuijper \& Johnstone, 2015; Leimar \& McNamara, 2015). The predictive power of environmental cues does not have to be perfect as anticipatory effects still evolved when environmental predictability was rather low. The costs of anticipatory effects influence the threshold of minimum predictability, since when there were no costs for any type of plasticity, anticipatory effects evolved even with minimal predictability. As usefulness of environmental cues in predicting the offspring environment decreases, less and less fitness benefit can be gained from anticipatory effects, and therefore only such anticipatory effects that have very low costs or no costs at all can evolve in rather unpredictable environments. Only a predictability of zero meant that there was not evolution of any kind of anticipatory effects. 


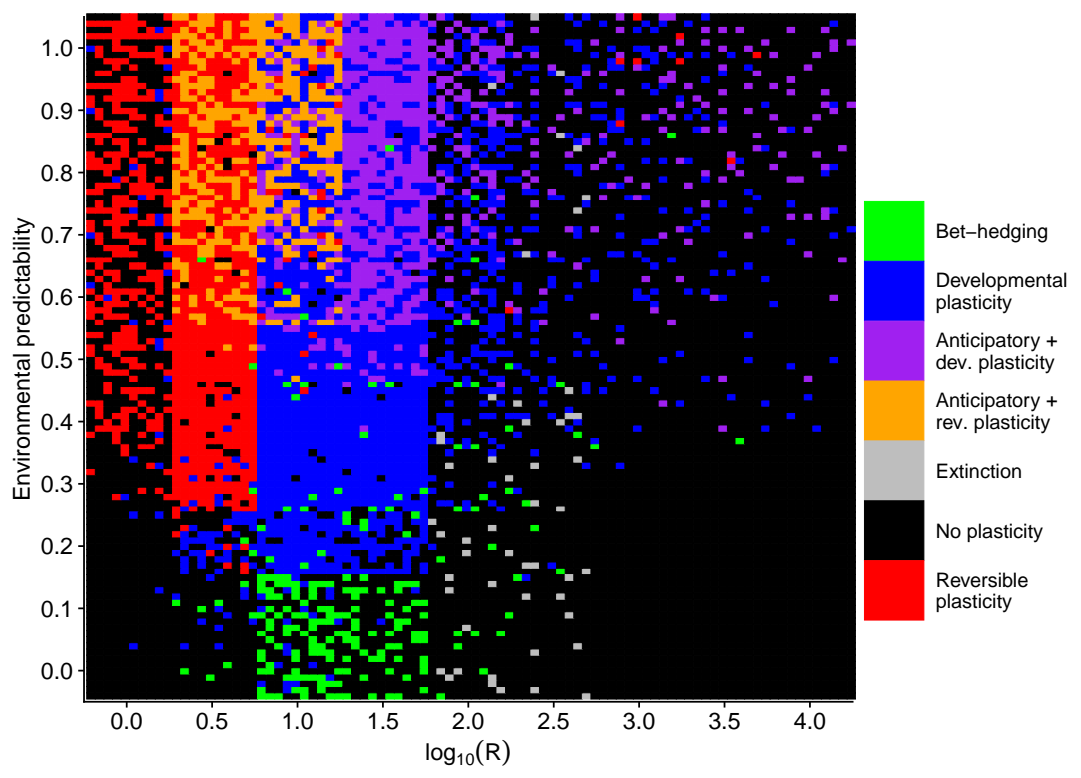

Figure 5: Sensitivity of results to genetic architecture. 100 replicate simulations were ran for each combination of $R$ and $P$, where parameter values for the random environmental effects, number of loci, number of chromosomes, mutation rate, and mutational effects were drawn randomly. Costs of plasticity were $k_{e}=k_{d}=0.02$ and $k_{a}=0.01$. Coloured tiles show which strategy evolved in the population or if it went extinct.

Anticipatory effects evolved only when frequency of environmental fluctuations was slow enough that cues per- 
seem to be generally weak (Uller et al., 2013). Most theory about the evolution plasticity in general assumes that there are some costs for plasticity (Callahan et al., 2008). However, while some studies have detected costs of plasticity (Relyea, 2002; Dechaine et al., 2007), in general costs of plasticity have been notoriously difficult to document in nature and we have a poor idea if we expect costs of anticipatory effects to be generally the same or somehow different. As fitness benefits of anticipatory effects are smaller then for within generation plasticity, it may be that that costs of anticipatory effects are an important component limiting their evolution.

Certain organisms seem to exhibit lot of anticipatory effects, such as nematodes (Baugh \& Day, 2020). This may reflect an experimental bias, as many people are studying these model organisms. However, a more likely explanation is that there is something in their ecology that favors the evolution of anticipatory effects. Environments change at different rates from the perspective of different organisms since generation times can be vastly different. What are fast environment changes for long lived multicellular organisms can be rather slow changes from the perspective of unicellular microbes. It may be, perhaps somewhat counter intuitively, that anticipatory effects can be more common in smaller organisms that are short lived. Moreover, the implication also is that organisms which have a long period between zygote formation and emergence of live offspring, such as mammals with long gestation periods, are expected to have less anticipatory effects than in organisms where there is a shorter time between zygote formation and emergence. The longer the time between setting the epigenetic marks themselves in gametes and offspring birth, the smaller the correlation between the environment of the offspring and the environment of the parent. In plants it is known that anticipatory effects are sometimes transmitted through seed but not by pollen (Herman et al., 2014; Wibowo et al., 2016), presumably because pollen can disperse over much longer distances, diminishing the correlation between parent and offspring environment. Unequal resetting of epigenetic marks in male and female gametes should apply as well to species where the time of gamete production and the time fertilization are decoupled, such as certain insects, reptiles, and birds that are capable of storing sperm for long periods of time (Boomsma et al., 2005; Holt \& Fazeli, 2016).

Another important ecological question is that how predictable real environments are from the perspective of organisms? Are there environmental cues that organisms can potentially use to predict future environments? The most obvious cues are related to changes in day length which does reliably predict seasons in high and low latitudes. Some environmental variables could also function as cues themselves, such as temperature. For example, high temperatures today can predict that temperature will also be high in the short term. Furthermore, there are some indications that many environmental variables are autocorrelated in nature (Halley, 1996), and that this autocorrelation often tends to be positive (Vasseur \& Yodzis, 2004; Ruokolainen et al., 2009), meaning that temporally close observations tend to be similar. Moreover, there seem to be differences in terrestrial and marine ecosystems, autocorrelation in terrestrial systems seems to be closer to zero, while marine ecosystems tend to have more positive autocorrelations (Vasseur \& Yodzis, 2004; Ruokolainen et al., 2009). It will be interesting to see, if anticipatory effects are more prevalent in marine organisms. Nevertheless, environments in nature tend to be at least somewhat autocorrelated, so there should be some 
opportunity for evolution of anticipatory effects.

In some empirical studies of epigenetically mediated anticipatory effects, the effects induced by the environment last for several generations. This has been particularly observed in nematodes (Baugh \& Day, 2020). The question remains are these multigenerational effects adaptive in nature, or rather a consequence of some developmental constraint? In the model used in this study it is difficult to see how multigenerational effects could be adaptive. As this would re-

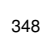


374 will undoubtedly be challenging. The model presented here could be tested experimentally by evolving eukaryotic microbes in environments that fluctuate at different frequencies and investigating whether the populations evolve plastic or anticipatory responses mediated by epigenetic changes.

\section{Acknowledgments}

This study was funded by an Academy of Finland Research Fellowship (no. 321584). I thank the Finnish CSC-IT Center for Science Ltd. for providing computational resources.

\section{References}

Agrawal, A.A., Laforsch, C. \& Tollrian, R. 1999. Transgenerational induction of defences in animals and plants. Nature 401: 60-63.

Baugh, L.R. \& Day, T. 2020. Nongenetic inheritance and multigenerational plasticity in the nematode C. elegans. eLife 9: e58498. doi:10.7554/eLife.58498. URL https : / / doi . org/10 . 7554 /eLife. 58498.

Boomsma, J.J., Baer, B. \& Heinze, J. 2005. The evolution of male traits in social insects. Annual Review of Entomology 50: 395-420. doi:10.1146/annurev.ento.50.071803.130416. URL https://doi.org/10.1146/annurev.ento.50.071803.130416. PMID: 15822204.

Botero, C.A., Weissing, F.J., Wright, J. \& Rubenstein, D.R. 2015. Evolutionary tipping points in the capacity to adapt to environmental change. Proceedings of the National Academy of Sciences 112: 184-189. doi: 10.1073/pnas.1408589111. URL http://www.pnas.org/content/112/1/184 .abstract.

Callahan, H.S., Maughan, H. \& Steiner, U.K. 2008. Phenotypic plasticity, costs of phenotypes, and costs of plasticity. Annals of the New York Academy of Sciences 1133: 44-66. doi:10.1196/annals.1438.008. URL https://nyaspubs.onlinelibrary.wiley.com/doi/abs/10.1196/annals.1438.008.

Dechaine, J.M., Johnston, J.A., Brock, M.T. \& Weinig, C. 2007. Constraints on the evolution of adaptive plasticity: costs of plasticity to density are expressed in segregating progenies. New Phytologist 176: 874-882. doi:10.1111/j.1469-8137.2007.02210.x. URL https://nph.onlinelibrary.wiley.com/doi/abs/10.1111/j.1469-8137.2007.02210.x.

Donelson, J.M., Munday, P.L., McCormick, M.I. \& Pitcher, C.R. 2012. Rapid transgenerational acclimation of a tropical reef fish to climate change. Nature Climate Change 2: 30-32. 
Duempelmann, L., Skribbe, M. \& Bühler, M. 2020. Small RNAs in the transgenerational inheritance of epigenetic information. Trends in Genetics 36: 203-214. doi:10.1016/j.tig.2019.12.001. URL https://doi.org/10.1016/j.tig.2019.12.001.

Durand, E., Tenaillon, M.I., Ridel, C., Coubriche, D., Jamin, P., Jouanne, S., Ressayre, A., Charcosset, A. \& Dillmann,

C. 2010. Standing variation and new mutations both contribute to a fast response to selection for flowering time in maize inbreds. BMC Evolutionary Biology 10: 2. URL https://doi .org/10.1186/1471-2148-10-2.

Ezard, T.H.G., Prizak, R. \& Hoyle, R.B. 2014. The fitness costs of adaptation via phenotypic plasticity and maternal effects. Functional Ecology 28: 693-701. doi:10.1111/1365-2435.12207. URL http://dx.doi.org/10.1111/1365-2435.12207.

Furrow, R.E. \& Feldman, M.W. 2014. Genetic variation and the evolution of epigenetic regulation. Evolution 68: 673-683.

Galloway, L.F. \& Etterson, J.R. 2007. Transgenerational plasticity is adaptive in the wild. Science 318: 1134-1136. URL http://www.sciencemag.org/cgi/content/abstract/318/5853/1134.

Greenspoon, P.B. \& Spencer, H.G. 2018. The evolution of epigenetically mediated adaptive transgenerational plasticity in a subdivided population. Evolution 72: 2773-2780. doi:10.1111/evo.13619. URL https://onlinelibrary.wiley.com/doi/abs/10.1111/evo.13619.

Halley, J.M. 1996. Ecology, evolution and 1/f-noise. Trends in Ecology \& Evolution 11: 33-37. doi:https://doi.org/10.1016/0169-5347(96)81067-6. $\quad$ URL http://www.sciencedirect.com/science/article/pii/0169534796810676.

Halligan, D.L. \& Keightley, P.D. 2009. Spontaneous mutation accumulation studies in evolutionary genetics. Annual Review of Ecology, Evolution, and Systematics 40: 151-172. doi:10.1146/annurev.ecolsys.39.110707.173437. URL http://dx.doi.org/10.1146/annurev.ecolsys.39.110707.173437.

Herman, J.J., Spencer, H.G., Donohue, K. \& Sultan, S.E. 2014. How stable 'should' epigenetic modifications be? insights from adaptive plasticity and bet-hedging. Evolution 68: 632-643.

224 Herman, J.J., Sultan, S.E., Horgan-Kobelski, T. \& Riggs, C. 2012. Adaptive transgenerational plasticity in an annual plant: Grandparental and parental drought stress enhance performance of seedlings in dry soil. Integrative and Comparative Biology 52: 77-88. doi:10.1093/icb/ics041. URL http://icb.oxfordjournals.org/content/52/1/77.abstract. 
Holeski, L.M. 2007. Within and between generation phenotypic plasticity in trichome density of Mimulus guttatus. Journal of Evolutionary Biology 20: 2092-2100.

Holt, W.V. \& Fazeli, A. 2016. Sperm storage in the female reproductive tract. Annual Review of Animal Biosciences 4: 291-310. doi:10.1146/annurev-animal-021815-111350. URL https://doi.org/10.1146/annurev-animal-021815-111350. PMID: 26526545.

Hoyle, R.B. \& Ezard, T.H.G. 2012. The benefits of maternal effects in novel and in stable environments. Journal of The Royal Society Interface 9: 2403-2413. doi:10.1098/rsif.2012.0183. URL http://rsif.royalsocietypublishing.org/content/9/75/2403.

Ivimey-Cook, E.R., Sales, K., Carlsson, H., Immler, S., Chapman, T. \& Maklakov, A.A. 2020. Transgenerational fitness effects of lifespan extension by dietary restriction in Caenorhabditis elegans. bioRxiv doi:10.1101/2020.06.24.168922. URL https://www.biorxiv.org/content/early/2020/06/24/2020.06.24.168922.

Jablonka, E., Oborny, B., Molnár, I., Kisdi, E., Hofbauer, J. \& Czárán, T. 1995. The adaptive advantage of phenotypic memory in changing environments. Philosophical Transactions of the Royal Society of London. Series B, Biological Sciences 350: 133-141.

Jablonka, E. \& Raz, G. 2009. Transgenerational epigenetic inheritance: prevalence, mechanisms, and implications for the study of heredity and evolution. Quarterly Review of Biology 84: 131-176.

Jobson, M.A., Jordan, J.M., Sandrof, M.A., Hibshman, J.D., Lennox, A.L. \& Baugh, L.R. 2015. Transgenerational effects of early life starvation on growth, reproduction and stress resistance in Caenorhabditis elegans. Genetics 201: 201-212. doi:10.1534/genetics.115.178699. URL http://www.genetics.org/content/early/2015/07/16/genetics.115.178699.abstract.

Kishimoto, S., Uno, M., Okabe, E., Nono, M. \& Nishida, E. 2017. Environmental stresses induce transgenerationally inheritable survival advantages via germline-to-soma communication in Caenorhabditis elegans. Nature Communications 8: 14031. URL http://dx.doi.org/10.1038/ncomms14031.

Kristensen, T.N., Ketola, T. \& Kronholm, I. 2018. Adaptation to environmental stress at different timescales. Annals of the New York Academy of Sciences doi:https://doi.org/10.1111/nyas.13974. URL https://nyaspubs.onlinelibrary.wiley.com/doi/abs/10.1111/nyas.13974.

Kronholm, I. 2017. Adaptive evolution and epigenetics. In: Handbook of epigenetics: The new molecular and medical genetics (T. Tollefsbol, ed), 2nd edn, pp. 427-438. Academic Press. 
Kuijper, B. \& Hoyle, R.B. 2015. When to rely on maternal effects and when on phenotypic plasticity?

Evolution

69: $\quad 950-968$.

URL https://onlinelibrary.wiley.com/doi/abs/10.1111/evo.12635. Biology pp. n/a-n/a. doi:10.1111/jeb.12778. URL http: / / dx . doi . org/10.1111/ jeb.12778.

Lachmann, M. \& Jablonka, E. 1996. The inheritance of phenotypes: An adaptation to fluctuating environments. Journal of Theoretical Biology 181: 1-9.

Lande, R. 2009. Adaptation to an extraordinary environment by evolution of phenotypic plasticity and genetic assimilation. Journal of Evolutionary Biology 22: 1435-1446.

Leimar, O. \& McNamara, J.M. 2015. The evolution of transgenerational integration of information in heterogeneous environments. The American Naturalist 185: E55-E69. doi:10.1086/679575. URL https://doi.org/10.1086/679575. PMID: 25674697.

Luna, E., Bruce, T.J., Roberts, M.R., Flors, V. \& Ton, J. 2012. Next-generation systemic acquired resistance. Plant Physiology 158: 844-853. doi:10.1104/pp.111.187468. URL http://www.plantphysiol.org/content/158/2/844.abstract.

Lynch, M. \& Walsh, B. 1998. Genetics and Analysis of Quantitative Traits. Sinauer Associates, Inc., Sunderland.

Marshall, J.D. \& Uller, T. 2007. When is a maternal effect adaptive? Oikos 116: 1957-1963.

Oomen, R.A., Kuparinen, A. \& Hutchings, J.A. 2020. Consequences of Single-Locus and Tightly Linked Genomic Architectures for Evolutionary Responses to Environmental Change. Journal of Heredity doi:10.1093/jhered/esaa020. URL https://doi.org/10.1093/jhered/esaa020. Esaa020.

Öst, A., Lempradl, A., Casas, E., Weigert, M., Tiko, T., Deniz, M., Pantano, L., Boenisch, U., Itskov, P., Stoeckius, M., Ruf, M., Rajewsky, N., Reuter, G., Iovino, N., Ribeiro, C., Alenius, M., Heyne, S., Vavouri, T. \& Pospisilik, J. 2014. Paternal diet defines offspring chromatin state and intergenerational obesity. Cell 159: 1352 - 1364. doi:http://dx.doi.org/10.1016/j.cell.2014.11.005. URL http://wWw.sciencedirect.com/science/article/pii/s0092867414014366.

Otto, S.P. \& Lenormand, T. 2002. Resolving the paradox of sex and recombination. Nature Reviews Genetics 3: 252-261. URL https://doi.org/10.1038/nrg761.

Prizak, R., Ezard, T.H.G. \& Hoyle, R.B. 2014. Fitness consequences of maternal and grandmaternal effects. Ecology and Evolution 4: 3139-3145. doi:10.1002/ece3.1150. URL http: / / dx . doi . org/10 . 1002 / ece3 . 1150. 
R Core Team 2019. R: A language and environment for statistical computing. R Foundation for Statistical Computing, Vienna, Austria. URL http: //www.R-project.org.

Rasmann, S., De Vos, M., Casteel, C.L., Tian, D., Halitschke, R., Sun, J.Y., Agrawal, A.A., Felton, G.W. \& Jander, G. 2012. Herbivory in the previous generation primes plants for enhanced insect resistance. Plant Physiology 158: 854-863. doi:10.1104/pp.111.187831. URL http://www.plantphysiol.org/content/158/2/854.abstract.

Rechavi, O., Houri-Ze'evi, L., Anava, S., Goh, W., Kerk, S., Hannon, G. \& Hobert, O. 2014. Starvation-induced transgenerational inheritance of small rnas in $C$. ele494 gans. $\quad$ Cell 158: 277 - 287. $\quad$ doi:https://doi.org/10.1016/j.cell.2014.06.020. URL http://www.sciencedirect.com/science/article/pii/s009286741400806X.

Rechavi, O. \& Lev, I. 2017. Principles of transgenerational small rna inheritance in Caenorhabditis elegans. Current Biology 27: R720 - R730. doi:https://doi.org/10.1016/j.cub.2017.05.043. URL http://www.sciencedirect.com/science/article/pii/s0960982217305791.

Rechavi, O., Minevich, G. \& Hobert, O. 2011. Transgenerational inheritance of an acquired small RNAbased antiviral response in C. elegans. Cell 147: 1248-1256. doi:10.1016/j.cell.2011.10.042. URL http://dx.doi.org/10.1016/j.cell.2011.10.042.

Relyea, R.A. 2002. Costs of phenotypic plasticity. The American Naturalist 159: 272-282. doi:10.1086/338540. URL https://doi.org/10.1086/338540. PMID: 18707379.

Ruokolainen, L., Lindén, A., Kaitala, V. \& Fowler, M.S. 2009. Ecological and evolutionary dynamics under coloured environmental variation. Trends in Ecology \& Evolution 24: $555-563 . \quad$ doi:https://doi.org/10.1016/j.tree.2009.04.009. $\quad$ URL http://www.sciencedirect.com/science/article/pii/s0169534709001864.

Salinas, S. \& Munch, S.B. 2012. Thermal legacies: transgenerational effects of temperature on growth in a vertebrate. Ecology Letters 15: 159-163.

Shama, L.N.S. \& Wegner, K.M. 2014. Grandparental effects in marine sticklebacks: transgenerational plasticity across multiple generations. Journal of Evolutionary Biology 27: 2297-2307. doi:10.1111/jeb.12490. URL http://dx.doi.org/10.1111/jeb.12490.

Slaughter, A., Daniel, X., Flors, V., Luna, E., Hohn, B. \& Mauch-Kani, B. 2012. Descendants of primed Arabidopsis plants exhibit resistance to biotic stress. Plant Physiology 158: 835-843. 
Uller, T., English, S. \& Pen, I. 2015. When is incomplete epigenetic resetting in germ cells favoured by natural selection? Proceedings of the Royal Society of London B: Biological Sciences 282: 20150682. doi: 10.1098/rspb.2015.0682.

Uller, T., Nakagawa, S. \& English, S. 2013. Weak evidence for anticipatory parental effects in plants and animals. Journal of Evolutionary Biology 26: 2161-2170. doi:10.1111/jeb.12212. URL https://onlinelibrary.wiley.com/doi/abs/10.1111/jeb.12212.

Vasseur, D.A. \& Yodzis, P. 2004. The color of environmental noise. Ecology 85: 1146-1152. doi:10.1890/02-3122. URL https://esajournals.onlinelibrary.wiley.com/doi/abs/10.1890/02-3122.

Wibowo, A., Becker, C., Marconi, G., Durr, J., Price, J., Hagmann, J., Papareddy, R., Putra, H., Kageyama, J., Becker, J., Weigel, D. \& Gutierrez-Marcos, J. 2016. Hyperosmotic stress memory in Arabidopsis is mediated by distinct epigenetically labile sites in the genome and is restricted in the male germline by DNA glycosylase activity. eLife $\mathbf{5}$ : e13546. doi:10.7554/eLife.13546. URL https://dx.doi.org/10.7554/eLife.13546. 
bioRxiv preprint doi: https://doi.org/10.1101/2020.09.30.321091; this version posted October 2, 2020. The copyright holder for this preprint (which was not certified by peer review) is the author/funder, who has granted bioRxiv a license to display the preprint in perpetuity. It is made available under aCC-BY-NC 4.0 International license.

\section{Supplementary Information}

${ }_{528}$ Supplementary Figures 
bioRxiv preprint doi: https://doi.org/10.1101/2020.09.30.321091; this version posted October 2, 2020. The copyright holder for this preprint (which was not certified by peer review) is the author/funder, who has granted bioRxiv a license to display the preprint in perpetuity. It is made available under aCC-BY-NC 4.0 International license.
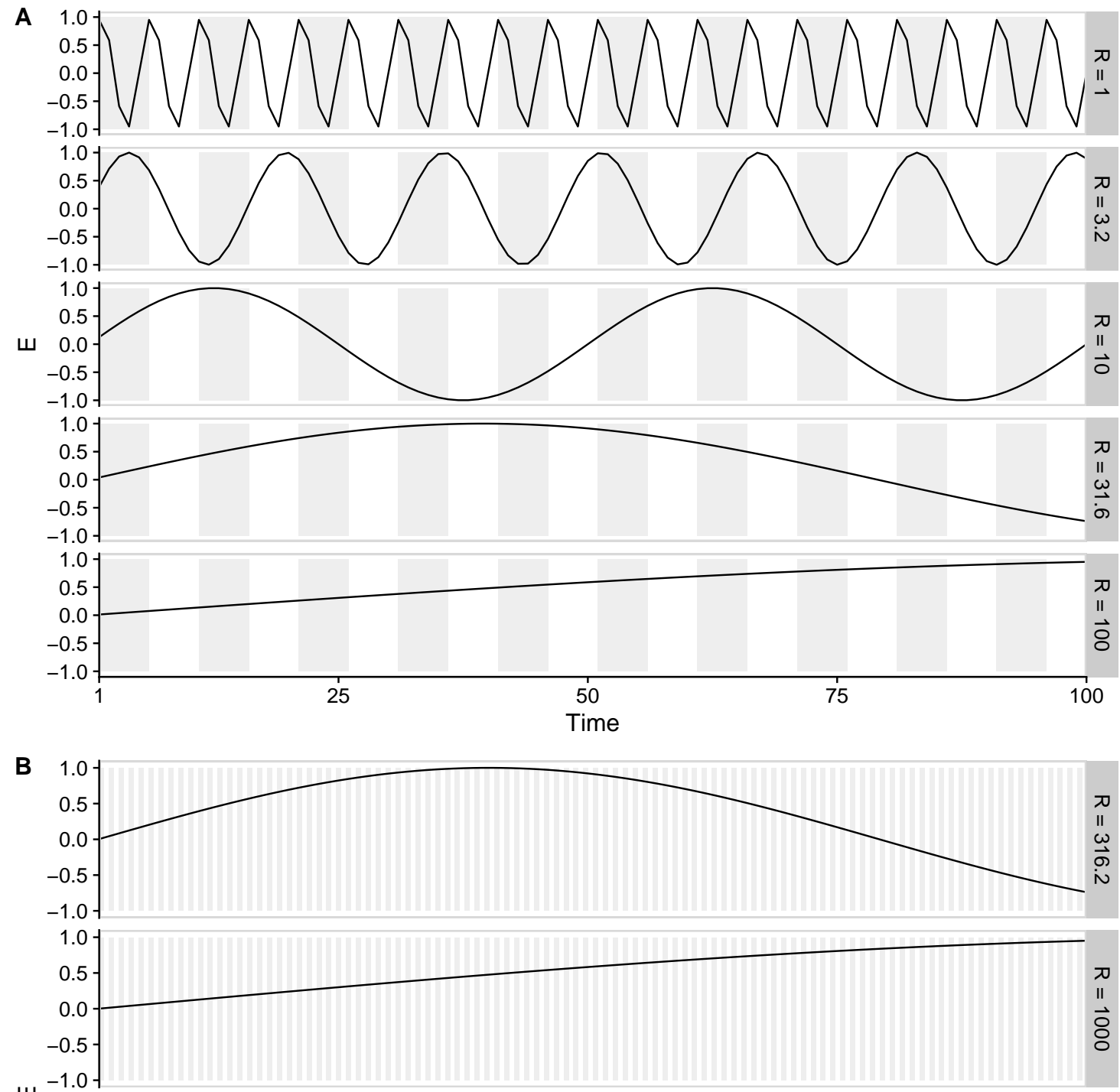

Ш

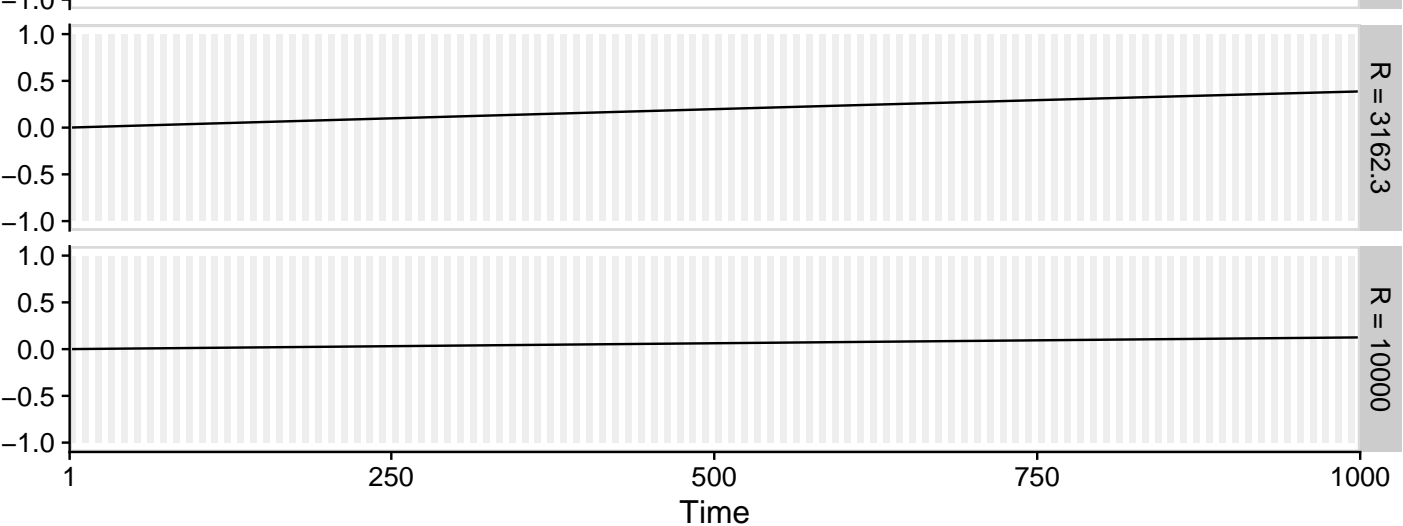

Figure S1: Illustration how the environment fluctuates with different values of $R$ used in the simulations. To illustrate different rates of environmental change, slower fluctuations are plotted on a different scale. A) Twenty generations are plotted. B) Two hundred generations are plotted. Each generation is five time steps long, as shown by alternation of shading. 

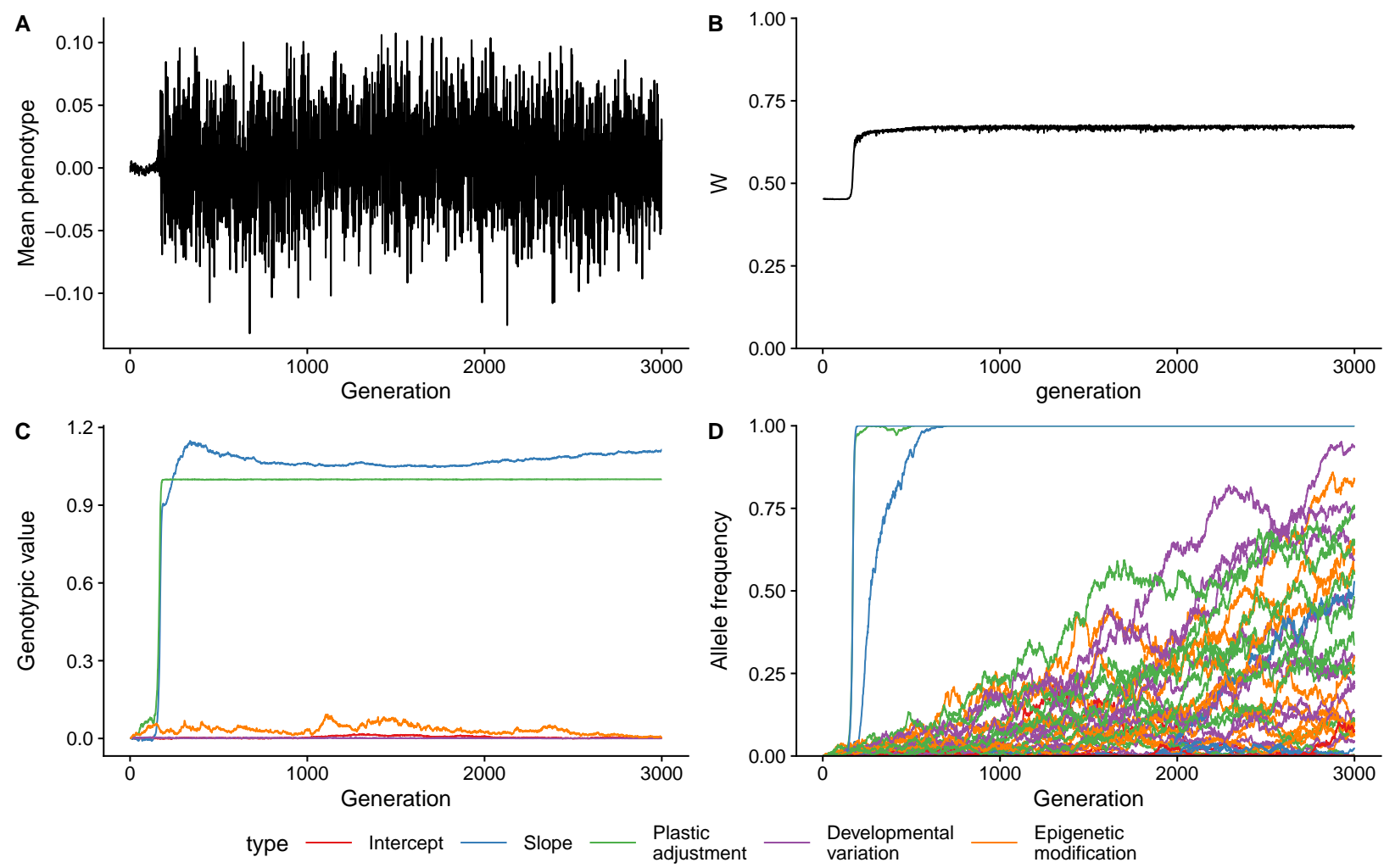

Figure S2: An example of how a single population evolves a strategy of reversible phenotypic plasticity. Simulation parameters were: $R=1, P=0.9, k_{d}=k_{e}=0.02$, and $k_{a}=0.01$. A) Phenotypic mean of the population for each generation. B) Population mean fitness for each generation. C) Genotypic values for reaction norm intercept, slope, probability of plastic adjustment, developmental variation, and probability of epigenetic modification for each generation. D) Allele frequencies of derived alleles at QTL controlling the genotypic values. Legend shows colours for types of genotypic values or loci. 

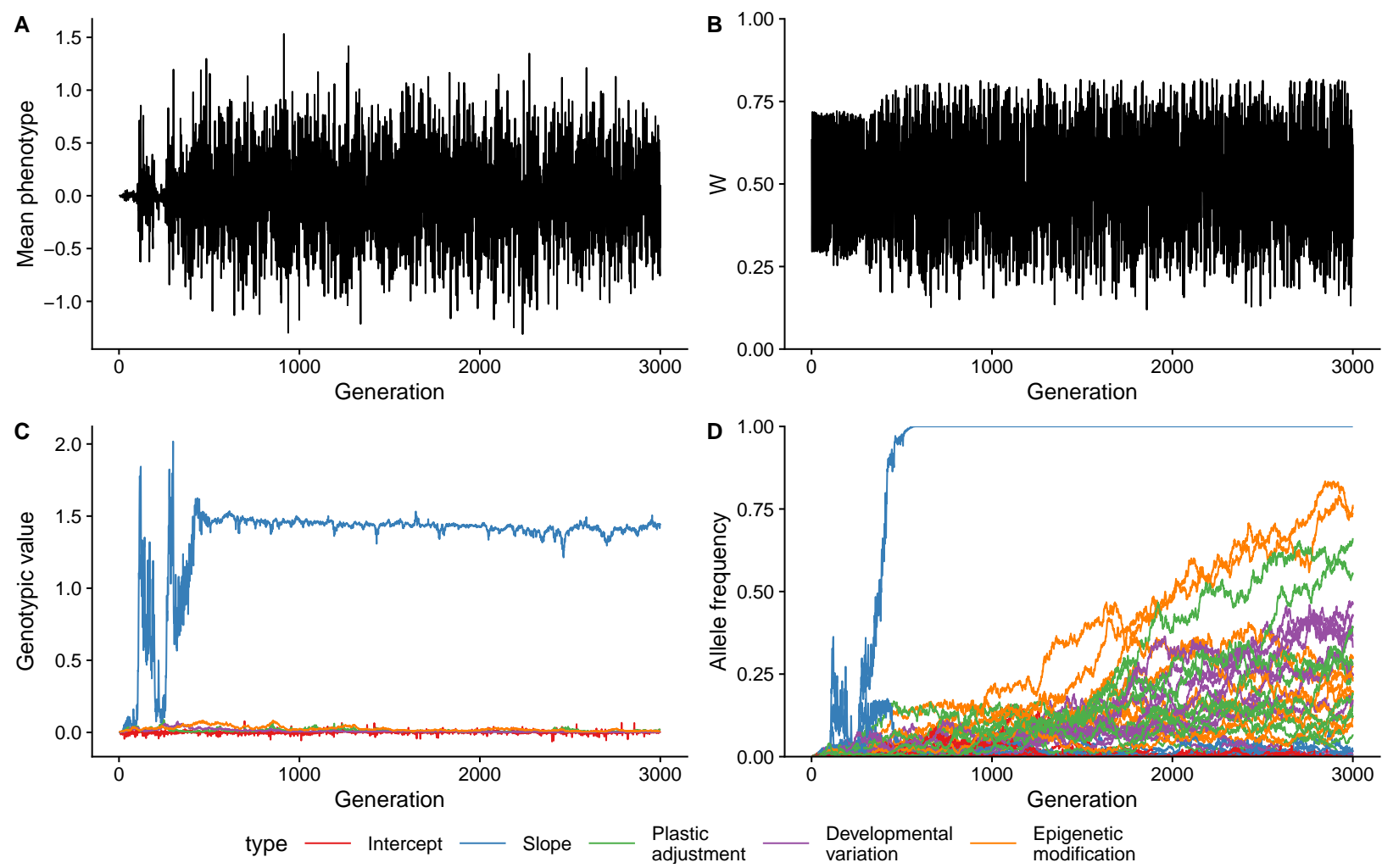

Figure S3: An example of how a single population evolves a strategy of developmental phenotypic plasticity. Simulation parameters were: $R=10, P=0.2, k_{d}=k_{e}=0.02$, and $k_{a}=0.01$. A) Phenotypic mean of the population for each generation. B) Population mean fitness for each generation. C) Genotypic values for reaction norm intercept, slope, probability of plastic adjustment, developmental variation, and probability of epigenetic modification for each generation. D) Allele frequencies of derived alleles at QTL controlling the genotypic values. 

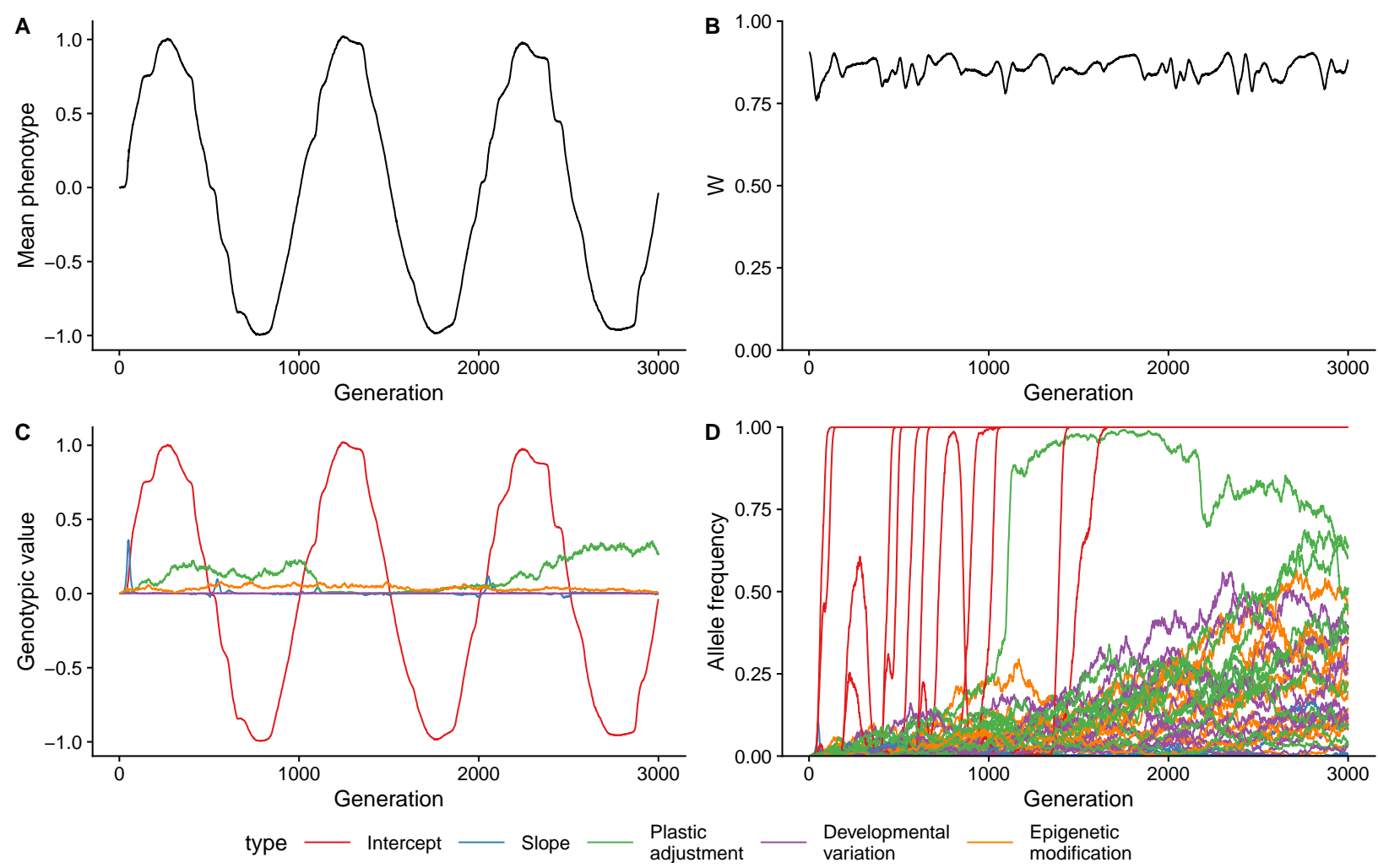

Figure S4: An example of how a single population evolves by tracking the environmental optimum by changing the reaction norm intercept. Simulation parameters were: $R=1000, P=0.9, k_{d}=k_{e}=0.02$, and $k_{a}=0.01$. A) Phenotypic mean of the population for each generation. B) Population mean fitness for each generation. C) Genotypic values for reaction norm intercept, slope, probability of plastic adjustment, developmental variation, and probability of epigenetic modification for each generation. D) Allele frequencies of derived alleles at QTL controlling the genotypic values. 

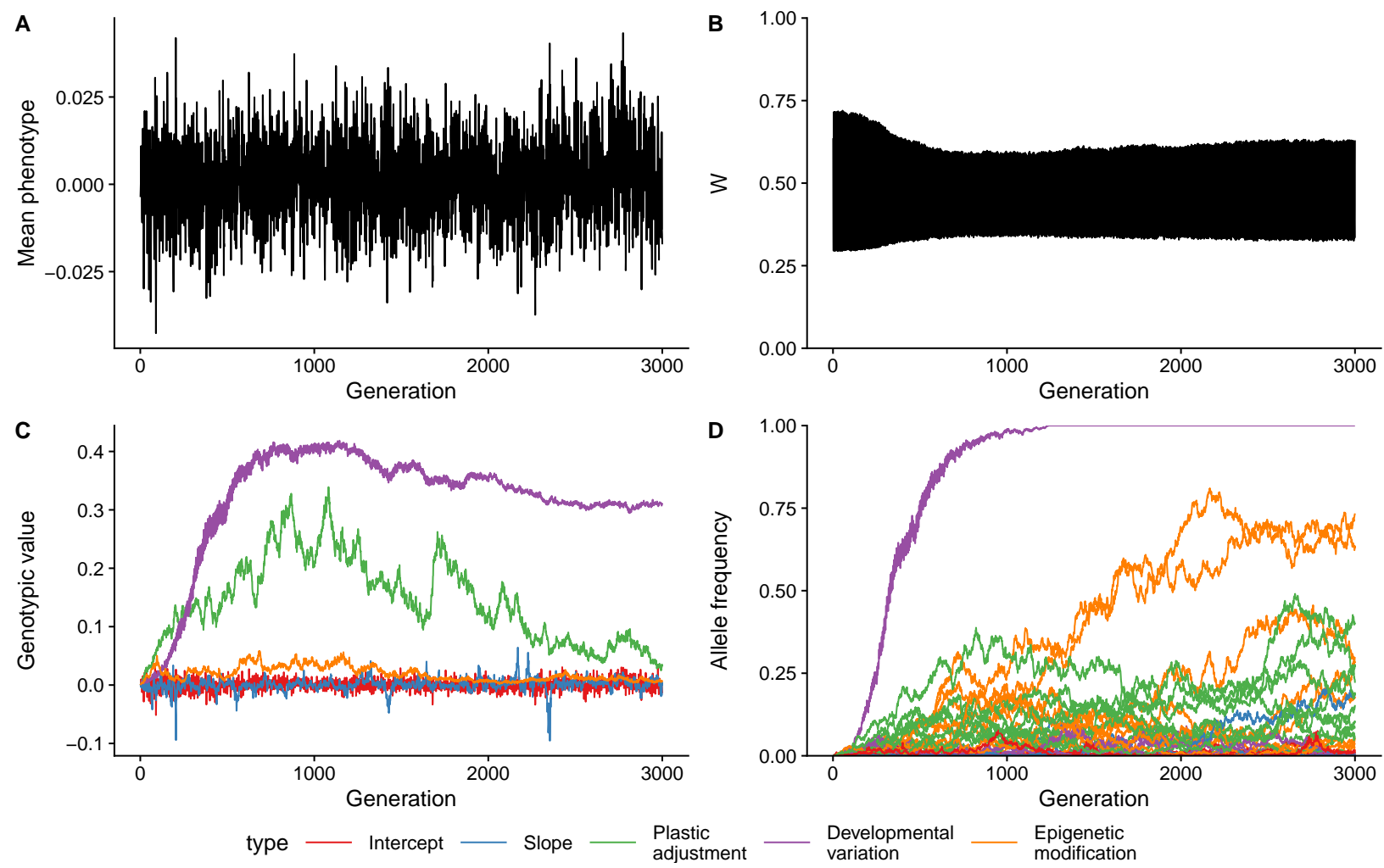

Figure S5: An example of how a single population evolves a strategy of diversifying bet-hedging. Simulation parameters were: $R=10, P=0, k_{d}=k_{e}=0.02$, and $k_{a}=0.01$. A) Phenotypic mean of the population for each generation. B) Population mean fitness for each generation. C) Genotypic values for reaction norm intercept, slope, probability of plastic adjustment, developmental variation, and probability of epigenetic modification for each generation. D) Allele frequencies of derived alleles at QTL controlling the genotypic values. 
bioRxiv preprint doi: https://doi.org/10.1101/2020.09.30.321091; this version posted October 2, 2020. The copyright holder for this preprint (which was not certified by peer review) is the author/funder, who has granted bioRxiv a license to display the preprint in perpetuity. It is made available under aCC-BY-NC 4.0 International license.
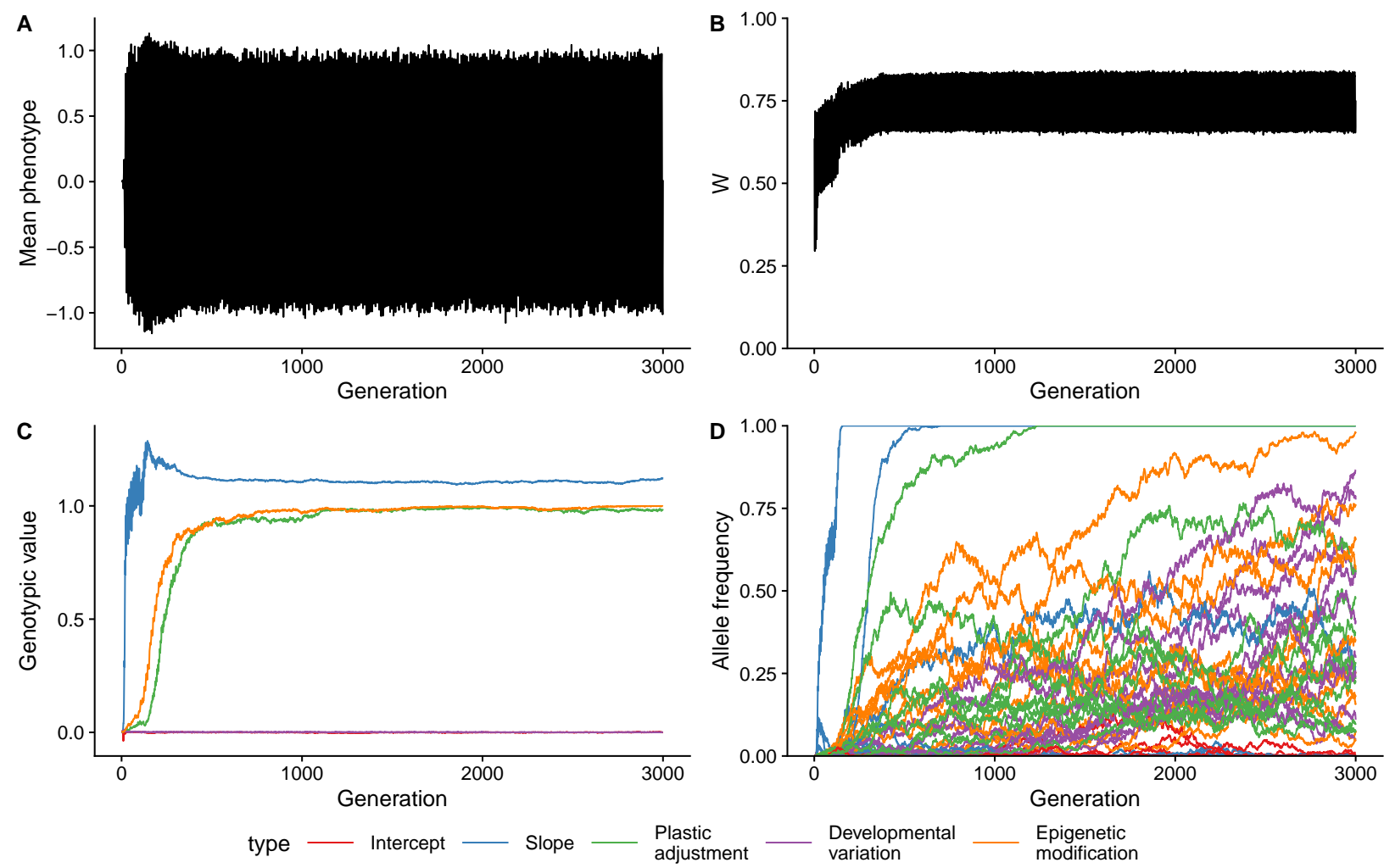

Figure S6: An example of how a single population evolves a strategy of reversible phenotypic plasticity and anticipatory effects via epigenetic modifications. Simulation parameters were: $R=10, P=0.9, k_{d}=k_{e}=0.02$, and $k_{a}=$ 0.01. A) Phenotypic mean of the population for each generation. B) Population mean fitness for each generation. C) Genotypic values for reaction norm intercept, slope, probability of plastic adjustment, developmental variation, and probability of epigenetic modification for each generation. D) Allele frequencies of derived alleles at QTL controlling the genotypic values. 

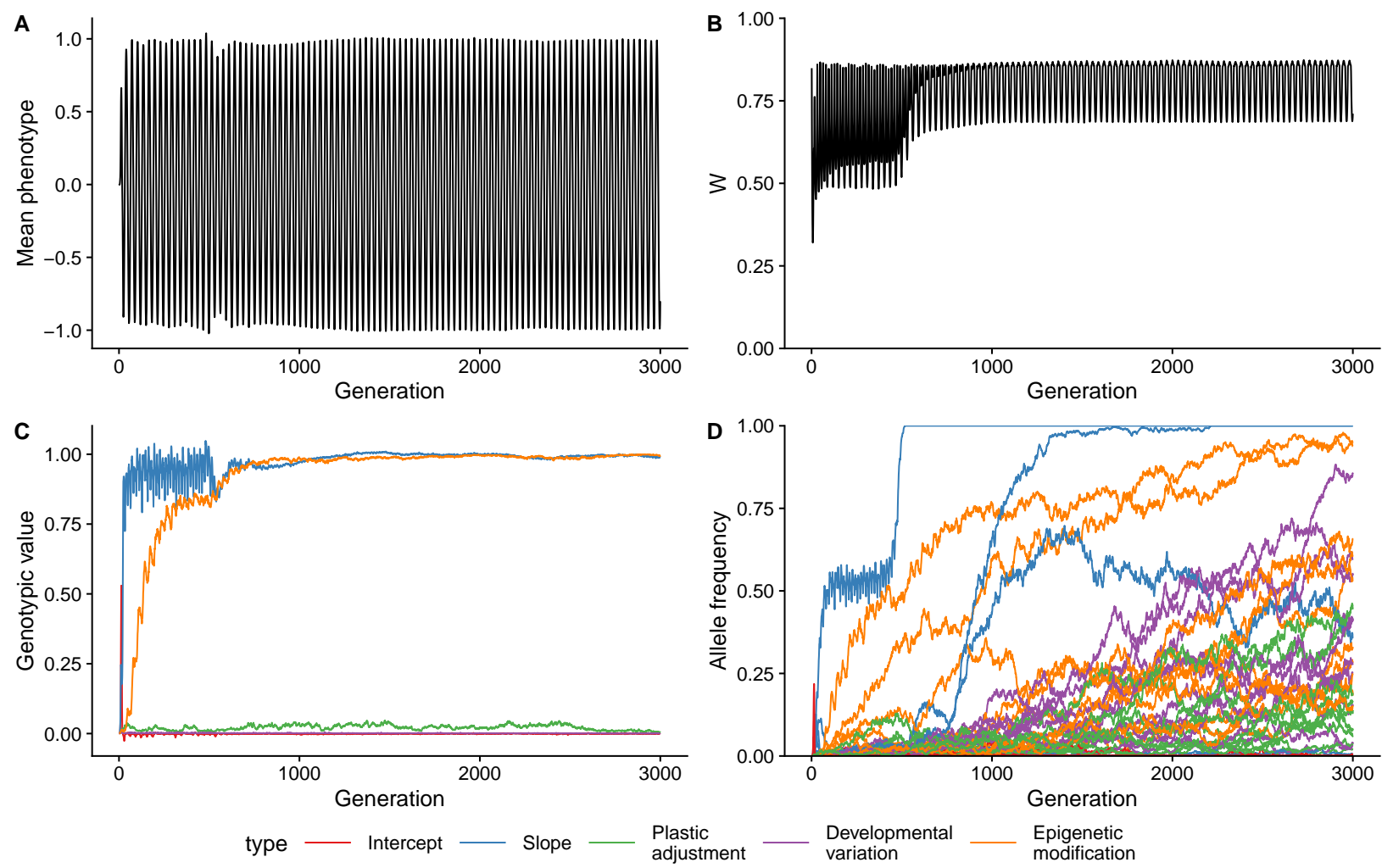

Figure S7: An example of how a single population evolves a strategy of developmental phenotypic plasticity and anticipatory effects via epigenetic modifications. Simulation parameters were: $R=31.6, P=1, k_{d}=k_{e}=0.02$, and $k_{a}=0.01$. A) Phenotypic mean of the population for each generation. B) Population mean fitness for each generation. C) Genotypic values for reaction norm intercept, slope, probability of plastic adjustment, developmental variation, and probability of epigenetic modification for each generation. D) Allele frequencies of derived alleles at QTL controlling the genotypic values. 

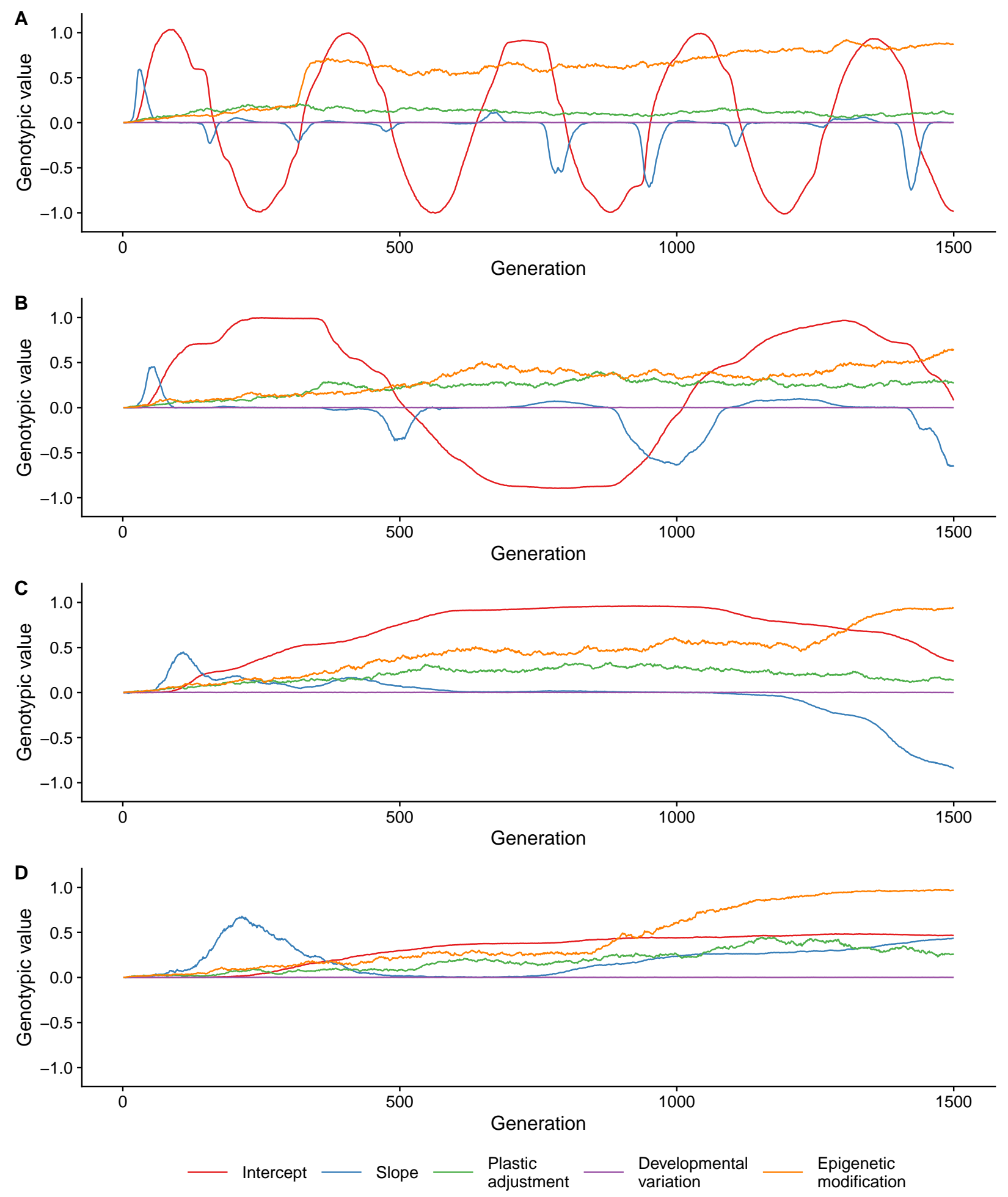

Figure S8: Examples of transient dynamics of plasticity and anticipatory effects punctuated by genetic assimilation. Genotypic values are shown for individual simulations runs. In all panels costs of plasticity are zero, $k_{d}=k_{e}=k_{a}=$ 0 , and $P=1$. A) $R=316.2$ B) $R=1000$ C) $R=3162.3$ D) $R=10000$ 